\title{
Implantación de marcapasos en niños
}

\author{
Oscar García P. ${ }^{1}$; Raùl Zilleruelo B. ${ }^{2}$; Oscar Gómez V. ${ }^{2}$ \\ Pacemaker implants in children
}

\begin{abstract}
Complications and lethality reated to aacemaker implants performed to 32 infants and children at a public cardiovascular center of Santiago, Chile, from december 1979 throughout december 1989 were retrospectively assessed Indications for pacemaker implants were complete congenital atrioventr cular block (n = 22; $69 \%$, and post-surgical arrhythmias i $n=10: 31 \%$ ). Replacement of pulse gevierator was necessary in 9 children after an average time of 5.5/12 years from the initial implant. Electrode fractures were detected in 4 cases of pacemaker's failure and raised stimulation threshold in one of such patients. Four patients of this series died along follow-up, only one death occurred in the immediate post-operatory period. The other 3 cases died several months after surgery due to causes not related to the pacemakers. The remaining 28 children are in good physical and emotiona! conditions.

(Key words: pacemakers, indicat ons, complications, lethality.)
\end{abstract}

En 1959, Elmquist y Senning, en Estocolmo, efectuaron la implantación del primer marcapasos cardíaco usando un electrodo epicárdico ${ }^{1}$. La estimulación eléctrica artificial, en el tratamiento de los trastornos del automatismo y la conducción del corazón, ha experimentado desde entonces una considerable evolución, debido a avances en la tecnología de los marcapasos: que han permitido desarrollar sistemas de estimula. ción más perfectos, y en los conocimientos clínicos, hemodinámicos y electrofisiológicos de los trastornos de la conducción cardíaca ${ }^{2,3}$.

Los niños son una minoría poco estudiada y mencionada, con respecto a los adultos, en el conjunto de candidatos a la instalación de marcapasos cardiacos. ${ }^{4.5}$. Las indicaciones para mat. capasos en la riñez se pueden agrupar en 6 criterios generales: bloqueo auriculoventricular completo (BAVC) con una o más crisis de StokeAdans: BAVC con frecuencias tan bajas que causan insuficiencia cardíaca; BAVC con frecuencia ventricular menor de $50 \cdot \mathrm{min}$, aun cuando no se acompañe de crisis de Stoke-Adams; BAVC postquirúrgico que no desaparece en 2 semanas; BAVC en recién nacidos con frecuencia ventricular menor de $50 \cdot \min$ o frecuencia auricular mayor de $150 \cdot$ min; enfermedad del nódulo sinu-

1. Becado de cardiología infantil. Hospital Luis Calvo Mackenna.

2. Servicio Cardjovascular, Hospital Luis Calvo Mackenna, Departamento de Pediatría. Escuela de Medicina, sede Oriente, Universidad de Chile. sal, con períodos de taquicardiabradicardia, ambas sintomáticas ${ }^{2,5-\vec{t}}$.

La revision que se ofrece a continuación se hizo con el propósito de describir las indicaciones, complicaciones y letalidad en la implantación de marcapasos electrónicos en niños atendidos en un centro pediátrico de referencia cardio. vascular, chileno.

\section{Pacientes y Método}

Se revisaron retrospectivamente los registros clínjcos de los niños a quienes les fueron implantados marcapasos elcctrónicos, en el Centro Cardiovascular del Hospital Luis Calvo Mackenna, entre los meses de diciembre de los años 1979 y 1989. In todos los casos se anotó scko, dagnóstico o causa de la indicación del marcapasos, enfermedad cardíaca asociada, edad de implantación, tipo de intervención, complicaciones, mortalidad inmediata $y$ taríía.

\section{Resultados}

Durante la década aludida se realizaron 68 intervenciones relacionadas con marcapasos en nifios, incluyendo primeras implantaciones, cambios de unidades generadoras y cambios de electrodos. De todas estas intervenciones se seleccionaron 32 casos en los que se había implantado marcapasos por primera vez. Diecinueve $(59 \%)$ eran varones. La causa de la indjcación fue BAVC congénito en 22 niños (69\%), BAVC postquirúrgico en $6 \operatorname{cosos}(19 \%$ ) y enfermedad del nódulo sinusal postquirúrgica en 4 pacientes $(12 \%)$. 
En 13 casos (40\%) se encontraron otros defectos cardiacos asociados. Tres pacientes con bloqueo A.V completo congénito tenian, respectivamente, defecto septal interauricular (DSIA) del tipo ostium secundum, canal auriculoventricular y fibroelastosis subendocárdica. En seis, los casos de pacientes cuyo BAVC ocurrió después de una intervención quirúrgica cardiovascular las anomalías cardiacas estaban cons. tituidas en dos casos por defecto septal interventricular (DSIV), un paciente con transposición de grandes arterias (TGA) y DSIV, otro con TGA corregida y DSIV, uno de estenosis mitral congénita y uno de in suficiencia mitral congénita. De 4 pacientes que después de operados sufrieron enfermedad del nódulo sinusal y fue necesario implantarles marcapasos, tres tenían TGA y uno drenaje venoso anómalo pulmonar total (DVAPT).

En el período que corresponde al estudio se hicieron 3.647 operaciones en este centro cardiovascular, de las cuales sólo $10(0,27 \%)$ originaron indicacjón posterior de marcapasos, como se describe en la tabla 1, entre ellas los únícos tres casos de TGA en los que se realizó un procedimiento de Mustard (desvío intraauricular de la sangre venosa hacia la válvula auriculoventricular pertinente) durante el periodo que abarca e] estudio. Las intervenciones operatorias asociadas con BAVC postquirúrgicos fueron cierres de DSIV con parche de dacrón en 4 niños (a ило de los cuales se efectuó, además, una operación de Mustard para corregir TGA) y reemplazos de válvula mitral por prótesis en dos pacientes. En los pacientes con enfermedad del nódulo sinusal postquirúrgica los procedimientos realizados fueron una operación de Senning (reinserción correctora de las arterias puimonar y aórtica) y dos de Mustard, respectivamente, en los casos de transposición de las grandes arterias y reconexión de las venas pulmonares en un paciente con DVAPT.

La edad de implantación de los marcapasos fluctuó entre los 2 días y los 16 años, distribuyéndose los pacientes en 2 recién nacidos $(6 \%), 8$ lactantes de 1 a 12 meses (25\%), 12 nifios de 1 a 5 años $(38 \%$ ) y 10 escolares de 5 a 6 años $(31 \%)$. Todos los marcapasos fueron instalados en pabellón de operaciones empleando toracotomía para insertar el electrodo epicárdico. En la mayoría se realizó incisión subcutánea abdominal para colocar la unidad generadora en un "bolsillo" abdominal del flanco o la fosa ilfaca izquier. da, pero en 3 casos la unidad generadora fue ubicada en posición torácica. En 2 casos se emplearon, por 2 y 8 semanas respectivamente, electrodos endocavitarios transitorios por vía venosa, procedimientos realizados en el pabellón de cateterismo, antes de colocar los epicárdicos defínitivos.

En el seguimiento de estos pacientes ha sido necesario cambiar la unidad generadora de im. pulsos, por haberse agotado, en 9 casos, en los cuales la duración promedio de su carga fue de $55 / 12$ años (límites 9 meses a $96 / 12$ años). Se detectaron fallas derivadas de los electrodos en 5 pacientes; ellas consistieron en aumento del umbral de estimulación en un niño, 5 meses des. pués de instalado el marcapasos, y fractura del electrodo en 4 niños, 8 ( 2 casos) y 14 meses después de la implantación. En el último niño la fractura del electrodo se repitió 5 meses después de haberlo cambiado.

En esta serie fallecieron cuatro pacientes. Una

Tabla 1

Necesidad de marcapasos definitivos según tipos de defectos cardíacos operados entre diciembre de 1979 y diciembre de 1989

\begin{tabular}{|c|c|c|c|c|}
\hline Defecto & & operaciones & $n \mathrm{MP}$ & \\
\hline $\begin{array}{l}\text { DVAPT } \\
\text { DSIV } \\
\text { TGA }\end{array}$ & $\begin{array}{l}135 \\
342 \\
149:\end{array}$ & $\begin{array}{lr}\text { Mustard } & 3 \\
\text { Senning } & 146\end{array}$ & $\begin{array}{l}1 \\
3 \\
3 \\
1\end{array}$ & (1 con TGA corregida) \\
\hline $\begin{array}{l}\text { Estenosis mitral } \\
\text { Insuficiencia mitral }\end{array}$ & $\begin{array}{r}16 \\
4\end{array}$ & & $\begin{array}{l}1 \\
1\end{array}$ & \\
\hline
\end{tabular}

DVAPT : drenaje venoso anómalo pulmonar total.

DSIV : defecto septal interventricular.

TGA : transposición de grandes artertas. 
niña de 43 días en que el BAVC estaba asociado con un canal auriculoventricular murió inmedia tamente después de colocado el marcapasos, comprobándose falla de captura. Otros tres niños murieron en tiempos alejados: una niña con TGA y DSIV fue operada a los 6 años con técrica de Mustard y reoperada a los 13 años por DSIV y estenosis pulmonar residuales; después de la reoperación sufrió enfermedad del nódulo sinusal, por lo que se le implantó uл marcapasos a la edad de 16 años, falleciendo 2 meses después por hernorragia pulmonar en relación a sepsis con falla orgánica múltiple; en su autopsia se encontró que, además, era portadora de carcinoma medular papilar tiroideo. Los otros 2 niños que fallecieron tardiamente tenian BAVC congénitos e ingresaron 1,5 y 2,5 meses respectivamente después del implante con evidencia de bronconeumonía, falleciendo ambos por dicha causa. En el primero de ellos, un varón de 3 meses de edad, se encontró fibroelastosis subendocárdica en la autopsia. Los 28 pacientes restantes se encuentran actualmente en control periodico en nuestro servicio; su capacidad funcional es I y muestran buen desarrollo físico y emocional.

\section{Discusión}

La indicación más común de marcapasos es la bradicardia sintomática, que puede deberse a BAVC, bloqueos de segundo grado o alteración de la función del nódulo sinusal ${ }^{1}$. E1 BAVC postquirúrgico ha sido descrito como la principal causa de implantaciones en nifios ${ }^{4,7,8}$, sibien la enfermedad del nodulo sinusal ha pasado a ser también una indicación frecuente ${ }^{2,11}$. En los casos postquirúrgicos se esperan dos semanas, con un electrodo epicárdico o endocárdico conectado a un generador externo, antes de indicar el marcapasos definitivo, pues en algunos casos el bloqueo desaparece en dicho tiempo. En estos pacientes el mecanismo fisiopatológico del bloqueo es la lesión traumática del haz đe Hiss al cerrar el DSIV o reemplazar la válvula mitral. La lesión del nódulo sinusal o de vías de conducción auricular es una complicación frecuente de la operaciôn de Mustard.

Los BAVC congénitos pueden ser aislados o asociarse con defectos cardíacos estructurales (esto último ocurre en 30\% de los casos). La transposición corregida de las grandes arterias es la anomalía más frecuentemente asociada, siendo otros el ventrículo único, el canal auriculoventricular, el DSIV aislado o en combi. nación con otras malformaciones cardíacas, la fjbroelastosis subendocárdica y las atresias tricuspídea y pulmonar. En este estudio la frecuencia de los bloqueos asociados a otros defectos fue de sólo $14 \%$, más baja que en otras series ${ }^{2}, 10-12$.

Por lo general, se observa cierta preferencia por utilizar electrodos epicárdicos, debido a las posibilidades de desplazamiento de los electrodos endocavitarios con el crecimiento y las consiguientes pérdidas de sensibilidad y aumento del umbral de estimulación del instrumento, como también los riesgos de trombosis venosa en estos $\operatorname{casos}^{4,5,10}$. Sin embargo, sistemas de anclaje en el ventrículo de mejor diseno nos han permitido el uso rutinario de electrodos endocavitarios en ritios mayores de 8 años. Cuando se emplean electrodos epicárdicos, la unidad generadora se coloca de preferencia en el abdomen, debido al tamaño de ella en relación al paciente, y en el flanco o la fosa ilíaca izquierda, para no obstaculizar vias de acceso ante la eventualidad de apendicectomía.

Si bien en el período comprendido en este estudio la frecuencia global de complicaciones secundarias a intervenciones quirúrgicas y conducentes a la indicación de marcapasos fue relativamente baja, en todos los casos de TGA en que se empleó el procedimiento de Mustard ocurrió alguna de ellas. Por este motivo, en nuestro centro se prefieren ahora las técnicas de Jatene o de Senning para la corrección de estos defectos, según el caso.

Entre las causas de fallas de los marcapasos se describen las derivadas de la unidad generadora (agotamiento de batería, infección, pérdida de conexión del electrodo y falla electrónjca) y las producidas por los electrodos (defecto de aistamiento, fractura, desplazamiento, posición inestable, disminución de la sensibilidad e interfe rencia, sea esta última por miopotenciales, aumento de amplitud de onda $T$ y $P$, señales electromagnéticas) $y$ aumento del umbral de estimulación ${ }^{1,3,4,7,13,14}$, siendo las más frecuentes las pocas observadas en nuestros pacientes. La duración actual de los MP es de 8 a 10 años $^{3,14}$, su tamaño ha sido reducido, como también su peso, de 35 a $40 \mathrm{~g}$. Actualmente se están usando, incluso, ingenios atómicos cuya duración y calidad son mejores aun que los más perfectos de los que utilizan baterías químicas de mercurio-zinc y litio. 
Esta experiencia confirma que los trastornos de la conducción cardiaca en niños, ya sea de origen congénito a postquirúrgico, tienen un tratamiento eficaz con los marcapasos, con un mínimo de letalidad y pocas fallas, todas solucionables al ser pesquisadas precozmente.

\section{Resumen}

El propósito de este estudio fue analizar las indicaciones de marcapasos en 32 niños, las complicaciones y la letalidad de éstos, en un centro cardiovascular metropolitano de referencia nacional, durante el período diciembre de 1979 a diciembre de 1989. La causa más frecuente de empleo de marcapasos fue el bloqueo auriculoventricular completo congénito (69\%), seguida por arritmias secundarias a procedimientos de cirugía cardíaca, los que incluyeron bloqueos auriculoventriculares y enfermedad del nódulo sinusal $(31 \%)$. Se cambió la unidad generadora en 9 niños, siendo la duración de ésta de 5 años 5 meses. En 4 pacientes el marcapasos falló por fractura del electrodo, y en uno por aumento del umbral de estimulación. Sólo un paciente falleció en periodo postoperatorio inmediato, con otros 3 fallecimientos alejados, debido a causas ajenas a) marcapasos. Los otros 28 pacientes restantes se encuentran en control en cardiología y muestran buen desarrollo físico y emocional.

(Palabras claves: marcapasos, indicaciones, complicaciones, mortalidad, cardiopatias congénitas.)

\section{Agradecimientos}

Los autores desean expresar sus agradecimientos al Dr. Leopoldo Romero, por la ayuda prestada en el uso del computador del Servicio Cardiovascular del Hospital Luis Calvo Mackenna.

\section{Referencias}

1. Kirklin, J.'Bradycardias. En: Cardiac Surgery, a Wiley Medical Publication. New York 1986; pp. $1347-1357$.

2. O'Callaghan, C.; Garcia, $C$.: Automatismo y conducción cardíacos. Editorial Institución Alfonso el Magnánimo. Valencia, Espafia 1984; pp. 377 . 382 y $653-660$.

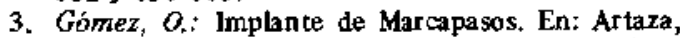
O; Zilleruelo, R. En: Manual de Cardiología y Cirugía Cardiovascular In fantil. Editorial Mediterráneo, Santiago, Chile 1989;pp. 277-279.

4. Dhooghe, $H$; Daenen, W.; Stolpoert, G.; Van der Hauwaert, $L$; $H$. de Guest.: Pacing in children. British Heart Journal, $1985 ; 53: \$ 41-\$ 46$.

5. Serwer, G.; Mericle, J.: Evaluation of pacemaker pulse generator and patient longevity in patients aged 1 day to 20 years. Ann J Cardiol 1987;59: $824-827$.

6. Fumon, S.: Young, D.: Cardiac pacing in children and adolescents. The Am J of Cardiol. April 1977; 39: $550-558$.

7. Benrey, J.; Gillette, P.; Nosrallah, A.; Hallman, G.: Permanent pacemaker implantation in infants, children and adolescents. Citculation 1976; 53: 245-248.

8. Hayes, D.; Holmes, D.: Maloney, J.; Neubauer, S.; Ritter, D.; Donielson, G.: Permanent endacardial pacing in pediatric patients. J Thorac Cardiovasc Surgery 1983; 85: 618-624.

9. Silka, M.; Manwill, J.; Kron, J.; MeAnulty, S.: Bradycardia mediated tachyarrythmias in congenital heart disease and responses to chronic pacing at physiologic rates. Am J Cardiol 1990; 65: 488-493.

10. Keith-Rowe-Vlad.: Heart disease in infancy and childhood. Mackillan Publishing Co., Inc. 1978; pp. 289-292.

11. Park, M.; Gutheroth, $W .:$ How to read pedittric ECGs. Year Book Medical Publishers, Inc. Second Edition, Chicago 1987; pp. 86-100.

12. Ross, B.: Congenital complete A-V block. En: Petiatric Clinics of North America, 1990; 37 : 69-77.

13. Serwer, G.; Mericle, J.; Amstrong, B.: Epicardial ventricular pacemaker electrode longevity in children. Am J Cardiol 1988; 61: 104-106.

14. Sandoe, E.; Sigmud, B, : Arthythmia, Diagnosis and Management, FACHMED, St. Gallen 1984; pp. 385-389. 